\title{
Editorial to selected papers from the XXI IMEKO World Congress 2015 and freely submitted articles
}

\author{
Paolo Carbone \\ University of Perugia, Italy
}

\begin{abstract}
Section: EDITORIAL
Citation: Paolo Carbone, Editorial to selected papers from the XXI IMEKO World Congress 2015 and freely submitted articles, Acta IMEKO, vol. 6, no. 1, article 1, April 2017, identifier: IMEKO-ACTA-06 (2017)-01-1
\end{abstract}

Section Editor: Paul Regtien, The Netherlands

Received April 19, 2017; In final form April 19, 2017; Published April 2017

Copyright: @ 2017 IMEKO. This is an open-access article distributed under the terms of the Creative Commons Attribution 3.0 License, which permits unrestricted use, distribution, and reproduction in any medium, provided the original author and source are credited

Corresponding author: Paolo Carbone, email: paolo.carbone@unipg.it

\section{Dear Reader,}

this year ACTA IMEKO will publish its 6th volume. This is a small but a rather important achievement for all the people involved in this venue. Let me take this occasion to thank all of them.

The first 6 papers in issue 6.1 originate from the 2015 World congress. The first paper is the paper by Michael Kobusch and Sascha Eichstädt who work at PTB. Their contribution is aimed at illustrating a problem in system identification in the area of mechanical measurements, so to achieve a model-based calibration of a strain gauge force transducer.

The following paper by Yukio Hiranaka, Shinichi Miura and Toshihiro Taketa takes into account the goal of determining the system input of a heater from measured data of room temperature. This leads to a backward simulator that tries to minimise processing time by taking into account the particular nature of the problem and also the usage of a forward model.

The paper by Sergey V. Muravyov, Irina A. Marinushkina, and Diana D. Garif covers the topic of inter laboratory comparison. It proposes a software to process experimental or simulated data by means of an aggregate preference method that, according to the authors, outperforms some other known rules that allow establishment of the reference value of a measurand.

The next paper by Michal Junek, Jiří Janovec and Petr Ducháček describes the method to measure the hardness of creep-resistant steel using ultrasound techniques to prove that the independence of the results obtained by such method from the Young's modulus of the specimen is not entirely correct.
The paper by Giovanni Betta et al. describes the issues associated with the metrological characterisation of 3D biometric face recognition systems. The problem of the performance evaluation of these systems is not yet fully addressed and this contribution shows how to compare experimental results obtained using various architectures under actual operating conditions.

The paper by Dragana Popovic Renella et al. contains an overview of commercially available teslameters used for measuring DC and AC magnetic flux densities. A thorough discussion is presented including the performance of such measurement instruments and more details are provided on gaussmeters based on 3-axis integrated Hall probes.

The next two papers in this issue were freely submitted by the authors. The paper by Catarina André et al. is in the area of measurements for the food industry. It discusses the design of statistical experiments to quantify the amount of freemonomeric isocyanates in aggregated cork stoppers. Experimental results are presented to validate their procedure.

The last paper in this issue by Dirk Röske et al. is the result of a collaboration among several National Metrological Institutes and describes the realization of a software tool for the calculation of uncertainty in force measurements. This research was developed through a research project in the European Metrology Research Programme.

Have a fruitful reading of the first issue of ACTA IMEKO in 2017 !

Paolo Carbone, Editor-in-Chief of ACTA Imeko 